\title{
Linking the Unsteady Force Generation to Vorticity for a Translating and Rotating Cylinder
}

\author{
P. Gehlert* and H. Babinsky ${ }^{\dagger}$ \\ University of Cambridge, Cambridge, United Kingdom, CB2 IPZ
}

\begin{abstract}
A boundary layer surface vortex sheet is utilized to investigate the development of the unsteady forces acting on a rotating and surging cylinder at a Reynolds number of $2 \times 10^{4}$. Planar particle image velocimetry and force balance measurements are employed to assess the flow field and force response. The boundary layer vortex sheet is categorized into non-circulatory and circulatory components. During acceleration the non-circulatory or added mass vortex sheet is successfully recovered from experimental data. This makes a decomposition of the forces, calculated only from particle image velocimetry measurements, possible. They compare well to force balance data. Additionally, the evolution of the boundary layer vortex sheet is assessed as the flow separates to form a vortical structure in the surrounding flow. When the influence of varying freestream velocity and rotation rate are removed, the surface vortex sheet at the location of separation varies little during vortex formation, even as the separation point moves along the surface of the cylinder.
\end{abstract}

\section{Nomenclature}

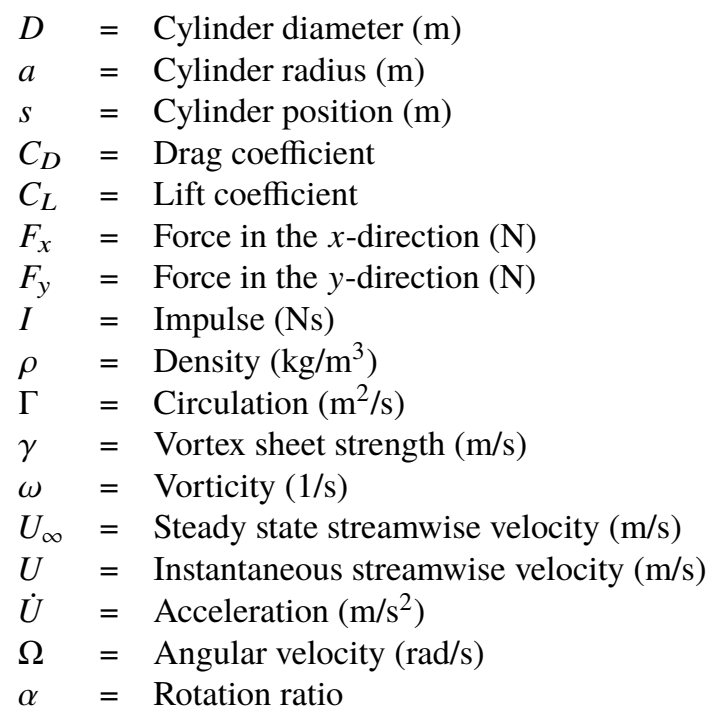

\section{Introduction}

$\mathrm{T}$ HE recent progression of technology has allowed for a significant development of micro-ariel vehicles (MAV). Some imitate the flight of insects or small birds whilst others resort to a fixed wing configuration. High gust ratios and turbulent fluctuations are common in their flight envelopes [1-3] causing both design approaches to experience large scale separations, either due to the unsteady kinematics of a flapping wing or through gust encounters. Extending the

*Ph.D Student, pg469@cam.ac.uk, Department of Engineering, University of Cambridge

${ }^{\dagger}$ Professor of Aerodynamics, Cambridge University Engineering Department, Associate Fellow AIAA 
area of focus, helicopter blades, water turbines or vertical axis wind turbines amongst others, also experience large changes of angle of attack, leading to unsteady force generation. The linear Küssner, Wagner and Theodorsen models, which assume small angles of attack and velocity perturbations, have severe limitations in such conditions. It has also been shown that in contrast to the assumptions made in these models, bound circulation is small in rapidly accelerating wings at large incidence, where flow separates at the leading edge to form a vortex [4]. Therefore, numerous studies have investigated the effect of the leading edge vortex (LEV) as well as on developing reduced low order models to predict the force response [5-7]. Being able to quickly model the forces, will allow for improved safety as well as provide the tools to design broader operating windows.

To better understand the origin of a force acting on a body, circulatory and non-circulatory force components have been identified. These have further been linked to respective circulatory and non-circulatory vortex sheets [8]. The latter was experimentally shown by Corkery \& Babinsky [9] to generate vorticity in the flow with zero total circulation for a thin flat plate. Separating the acting forces into their components is useful when building low order models, as the contributions can be modelled independently and superimposed at the end to yield the total force.

In an effort to inform the development of unsteady low order models, this paper seeks to evaluate the evolution of the surface vortex sheet around a generalised body of finite thickness. To this end, a rotating and translating circular cylinder is used. In particular, we aim to investigate the vortex sheet development at the point of flow separation to gain insight into the circulatory force production. The non-circulatory force component will further be recovered using the corresponding non-circulatory vortex sheet.

The paper will begin by presenting an experimental set-up which allows the measurement of the complete flow field around a translating and rotating cylinder. To recover the surface vortex sheet components, the methodology proposed by Eldredge [8], Graham et al. [10] and Corkery \& Babinsky [9] is extended to a general case and demonstrated in an applied experimental setting. This consequently allows the forces and importantly the force components to be recovered purely from particle image velocimetery (PIV); as long as two-dimensional flow can be assumed.

\section{Methodology}

\section{A. Experimental Set-Up}

The tests are conducted in the towing tank facility at the University of Cambridge Engineering Department. The tank is $10 \mathrm{~m}$ long and $1 \mathrm{~m}$ wide and the water depth is $0.8 \mathrm{~m}$ for all test cases. The cylinder is vertically mounted to a force balance which is connected to a servo motor driven carriage. Force measurements and particle image velocimetry data are simultaneously acquired.

A qualitative sketch of the experimental set-up is shown in figure 1 The cylinder is $0.06 \mathrm{~m}$ in diameter and consists of a hollow carbon fibre tube with a span of $0.48 \mathrm{~m}$. Due to the presence of the skim plate, the effective aspect ratio of the cylinder is 16 . The effective aspect ratio is further increased through the use of an endplate at the far side of the cylinder, as seen in figure 2. Inside this cylinder, a load bearing, hollow, aluminium tube houses a carbon fibre drive shaft. The aluminium tube is attached to the bottom of the force balance and has two bearings on either end, on which the outer cylinder sits. An EC motor, fitted with a digital encoder to measure the angular velocity, is mounted to the force balance and connects to the drive shaft via a rotary coupling. The endplate mounted to the bottom of the cylinder is made out of clear, see-through acrylic and does not block the optical access. Further care was taken to ensure that the region directly around the cylinder was never obscured by the endplate as seen by the two cameras. A schematic illustration of the cylinder assembly is shown in figure 2. 


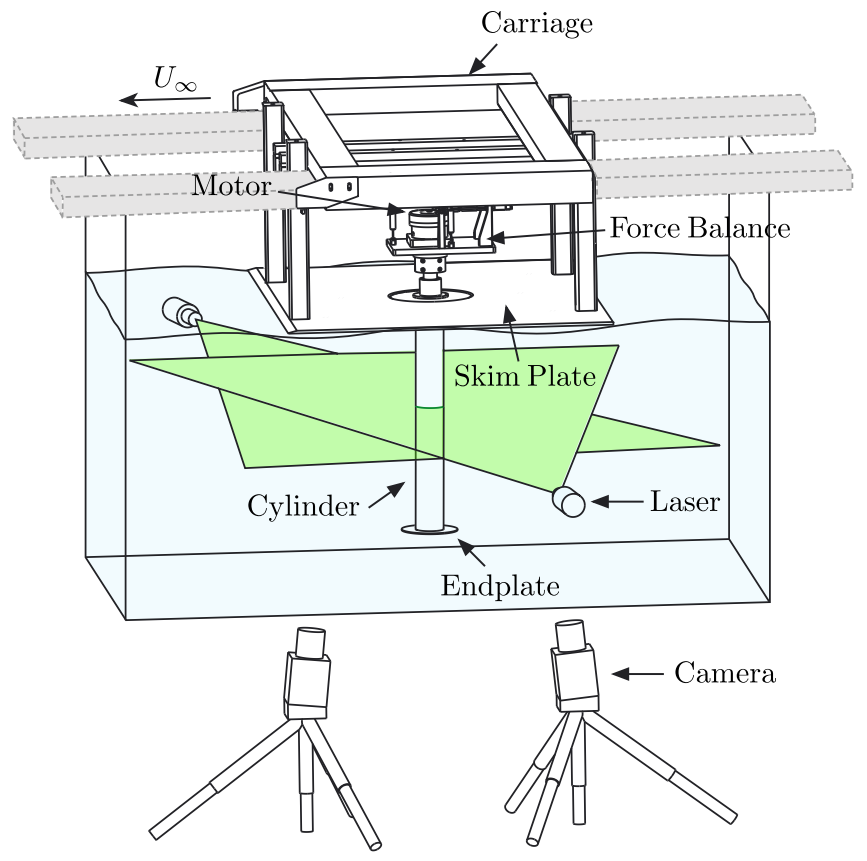

Fig. 1 Experimental set-up.

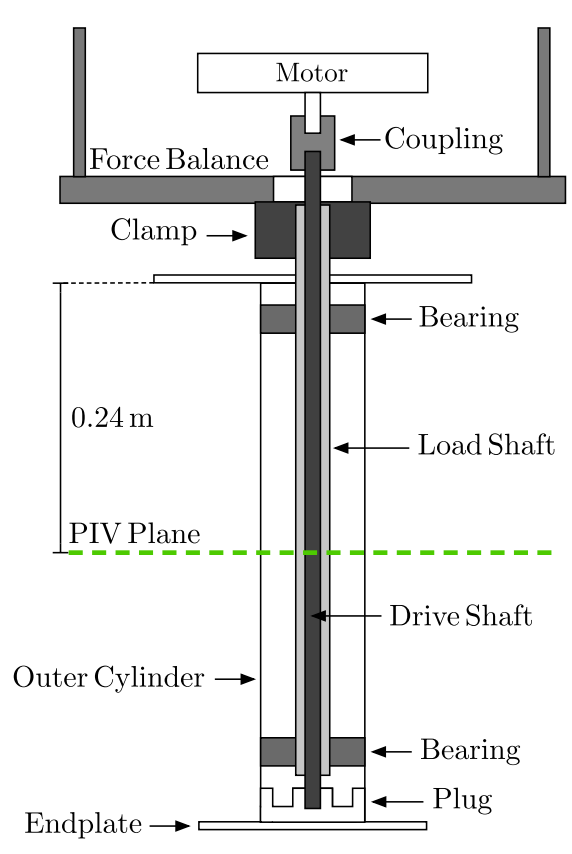

Fig. 2 Cylinder assembly sketch.

\section{B. Cylinder Kinematics}

The two cases that are used to explore the force response are schematically represented in figure 3 and a summary of the kinematic parameters is provided in table 1

Case 1

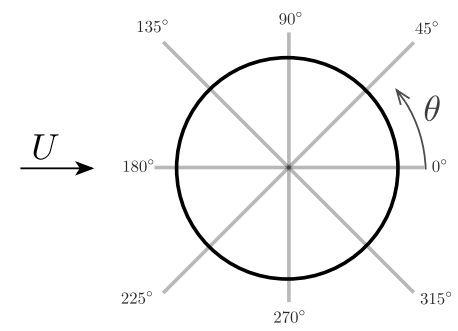

Case 2

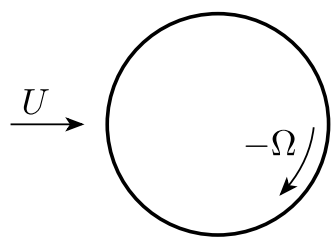

Fig. 3 Experimental configurations. The angle definition around the cylinder is also indicated.

\section{Case 1 - Surging Cylinder}

The cylinder begins to translate from a stationary start. It accelerates over a distance of two or three diameters after which it continues to translate at a constant velocity of $0.43 \mathrm{~ms}^{-1}$. This leads to a steady state Reynolds number, $R e$, of $2 \times 10^{4}$.

\section{Case 2 - Surging and Spinning Cylinder}

The cylinder simultaneously begins to translate and rotate from a stationary start. It follows the same translational profiles as outlined in Case 1. Case 2 is repeated for a slow and a fast rotating case, where the cylinder is spinning at 137 and 411 revolutions per minute (RPM), respectively. This results in a rotation ratio, $\alpha=\frac{\Omega_{\infty} r}{U_{\infty}}$, of 1 and 3 . A constant angular velocity, $\Omega_{\infty}$ is reached within $s / D=0.15$ and $s / D=0.31$ when $\alpha=1$ and $\alpha=3$. 


\begin{tabular}{ccccccc}
\hline \hline & $\boldsymbol{\alpha}$ & $\mathbf{R P M}$ & $\boldsymbol{U}_{\boldsymbol{\infty}}\left(\mathbf{m s}^{-\mathbf{1}}\right)$ & $\boldsymbol{R e}$ & $\boldsymbol{s}_{\mathrm{acc}} / \boldsymbol{D}$ & $\boldsymbol{s}_{\boldsymbol{\Omega}_{\infty}} / D$ \\
\hline \multirow{2}{*}{ Case 1 } & 0 & 0 & 0.43 & $2 \times 10^{4}$ & 2 & - \\
& 0 & 0 & 0.43 & $2 \times 10^{4}$ & 3 & - \\
\hline \multirow{2}{*}{ Case 2 } & 1 & 137 & 0.43 & $2 \times 10^{4}$ & 2 & 0.15 \\
& 3 & 411 & 0.43 & $2 \times 10^{4}$ & 2 & 0.31 \\
\hline \hline
\end{tabular}

Table 1 Summary of the test cases. $s_{\text {acc }}$ represents the acceleration distance and $s_{\Omega_{\infty}}$ the distance required to reach terminal angular velocity, $\Omega_{\infty}$.

An example of the kinematic trace of the cylinder including the measured velocity, acceleration and RPM for Case 2 when $\operatorname{Re}=2 \times 10^{4}$ and $\alpha=3$ averaged over 5 runs is reported in figure 4 . The ideal and experimental velocity trace match well throughout the motion, with some vibrations being noticeable during the steady state region, as seen in figure 4a. Initially, the real acceleration overshoots and at the end of the acceleration phase decays to zero over a prolonged distance, compared to the ideal case, as presented in figure $4 \mathrm{~b}$. The rotation rate of the cylinder matches well to the ideal case, figure $4 \mathrm{c}$. however, takes a finite distance before the steady state region is reached. The corresponding distance for each test case is indicated in table 1.

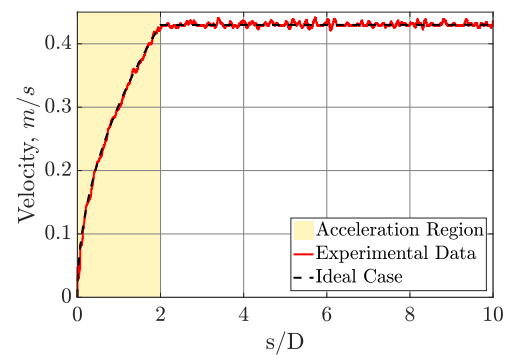

(a) Velocity.

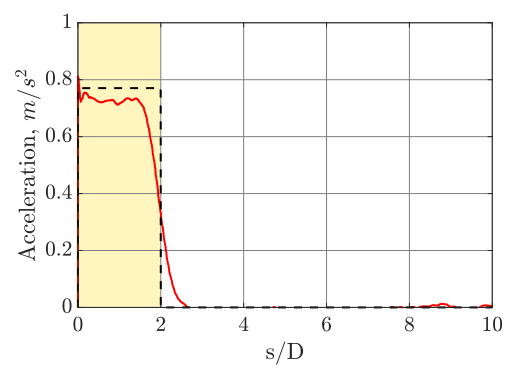

(b) Acceleration.

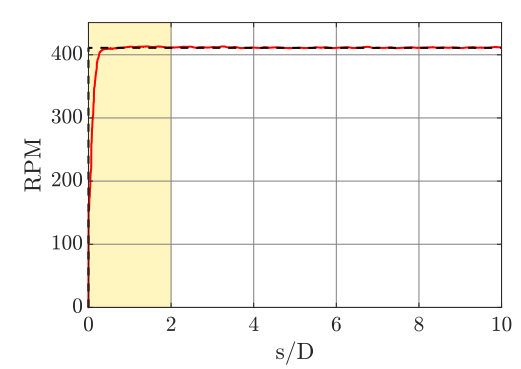

(c) RPM.

Fig. 4 Kinematic profile of the cylinder. Case 2, $R e=2 \times 10^{4}, \alpha=3$.

\section{Force Measurements}

The forces acting on the cylinder are recorded with a Flow Dynamics two component load cell. It allows force measurements between $\pm 50 \mathrm{~N}$ with a resolution of $0.01 \mathrm{~N}$ and is calibrated in situ to obtain a representative force curve slope. The carriage position is obtained using an electro-optical sensor with a resolution of $1 \mathrm{~mm}$. The velocity is determined through numerical differentiation of the position data, whilst the acceleration is measured by an ADXL335 3-component micro-electromechanical accelerometer mounted to the carriage. All measurements are sampled at a rate of $3 \mathrm{kHz}$.

The force data is averaged over 5 runs and a zero phase shift averaging window is applied to the data. A comparison between a typical run and the final filtered average is shown in figure 5 . The test case with the highest rotation rate and surge velocity is shown, $\alpha=3$ and $R e=2 \times 10^{4}$. A conservative estimate of the random error for a typical run is approximately $1 \%$ relative to the peak mean force, thus indicating good repeatability between runs.

The inertial force component is removed from the measured data to isolate the aerodynamic force acting on the cylinder. This is done by determining the inertial force, $F_{i}$, and subtracting it from the total measured force. $F_{i}$ is computed using the instantaneous acceleration, $\dot{U}$, and the mass of the unsupported cylinder and force balance assembly $m_{u s}$;

$$
F_{i}=m_{u s} \dot{U}
$$




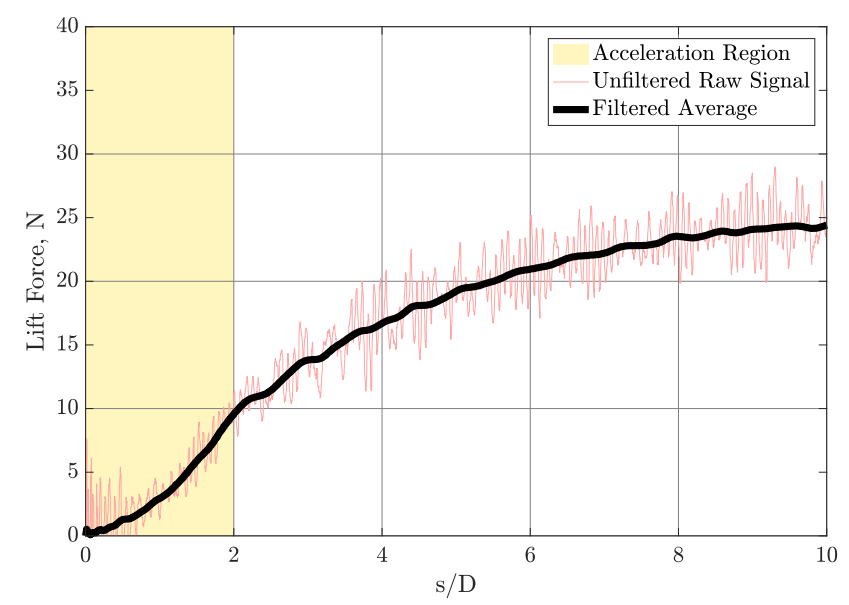

Fig. 5 Lift force history and filtered average, $R e=2 \times 10^{4}, \alpha=3$.

The variation of the translation velocity has an associated uncertainty of $1 \%$. Density variations due to a change in temperature of the water are found to be too small to contribute to the uncertainty of the force coefficients. Further assessing the uncertainty of $m_{u s}$ and the accelerometer leads to a total uncertainty of approximately $3 \%$ to be associated with the final force coefficients.

\section{Particle Image Velocimetry}

PIV measurements are conducted in an arrangement similar to that illustrated in figure 1 An Nd:YLF $527 \mathrm{~nm}$ laser is used to illuminate Titanium Dioxide particles that are suspended in water and two high speed Phantom M310 cameras are used for the image acquisition. Each camera has a resolution of $1280 \times 800$ pixels. The sampling rate is adjusted between $900 \mathrm{~Hz}$ and $1300 \mathrm{~Hz}$ and all images are acquired at the midspan and post-processed using the commercial LaVision Flowmaster 2D system. The PIV measurements are averaged over 5 to 6 runs and the size of the adaptive interrogation window during its final pass is $16 \times 16$ pixels with an overlap of $50 \%$.

To capture the full flow field around the cylinder, without any shadow regions, a dual light sheet is employed. Initially, the laser beam is split into two separate beams after which they are shone into the test section from either side. The laser optics are offset in the $x$-direction to create a region of approximately 5 diameters where all shadows are eliminated. A top view of this arrangement is illustrated in figure 6.

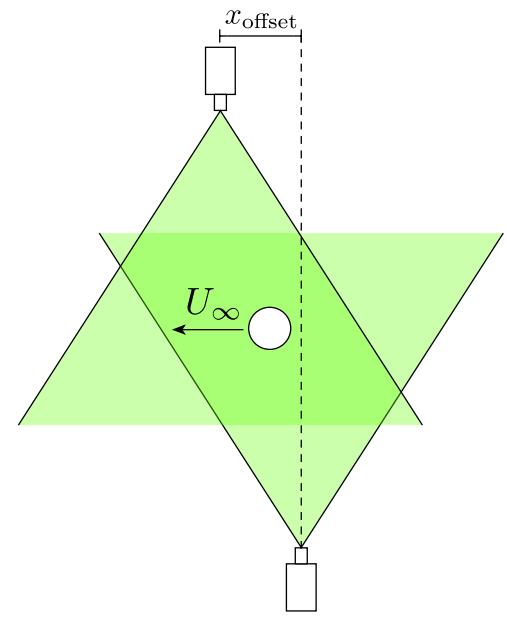

Fig. 6 Top view of the laser arrangement. 
The first camera is positioned behind the cylinder and the second is located in front of it. This allows the flow field on the rear of the cylinder to be viewed by the first camera, whilst the second camera has optical access to the region in front of the cylinder. Both PIV frames are then post-processed independently, using a single optical calibration, after which the resulting vector field is stitched together. This provides one continuous vector field containing data on either side of the cylinder.

Planar PIV recovers the velocity field on a given two dimensional plane. If the cameras are exactly normal to this plane, the velocity vectors aligned with the plane can be fully recovered, even if there is a normal velocity component, $w$. If the cameras are at an angle $\varphi$ to this plane then the particle motion is incorrectly resolved. The error in the velocity component is

$$
u_{\text {error }}=\cos \psi \frac{w}{\sin \psi}=w \cot \psi \mathrm{ms}^{-1},
$$

where $\varphi$ is the angle between the camera and the PIV plane.

A vertical PIV plane, normal to the direction of motion located at $s / D=2$, is used to find $w$ for the individual cases. $|w|$ is averaged over four runs and 100 frames for each case. A conservative estimate of the $u_{\text {error }}$ is obtained by extracting the maximum absolute vertical velocity and noting $\varphi=85^{\circ}$. The obtained percentage error is below $0.5 \%$. Together with the peak locking error, which dominates the root mean square (RMS) velocity error [11], the total measurement error is estimated to be below $3 \%$. To remove spurrious erroneous velocity vectors a median filter [12] is used to remove vectors if their RMS is more than double that of their neighbours.

\section{E. Impulse Method}

The forces acting on a body can be evaluated from the vorticity in the flow field using the impulse approach proposed by $\mathrm{Wu}$ [13]. To achieve this, the complete vorticity field around the body must be captured, including the starting vortex. In practice, this is often difficult to achieve because the vorticity in the boundary layer must be resolved at the same time. Increasing the field of view makes it possible to measure all of the vorticity, however, it reduces the resolution of the obtained velocity vectors. This results in greater regions of missing velocity data close to the cylinder surface and thus omits vorticity in the boundary layer. Figure 7 illustrates the complete flow field that needs to be measured, including the starting vortex, vorticity in the boundary layer and any free vorticity.

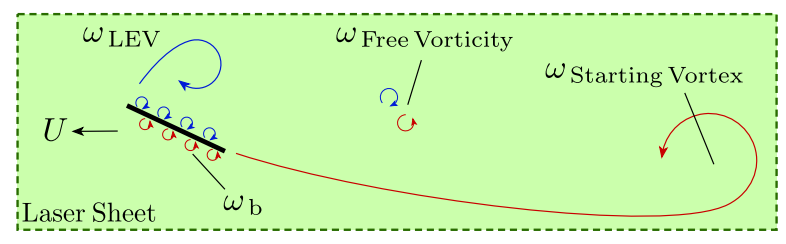

Fig. 7 Complete vorticity field for a surging flat plate wing.

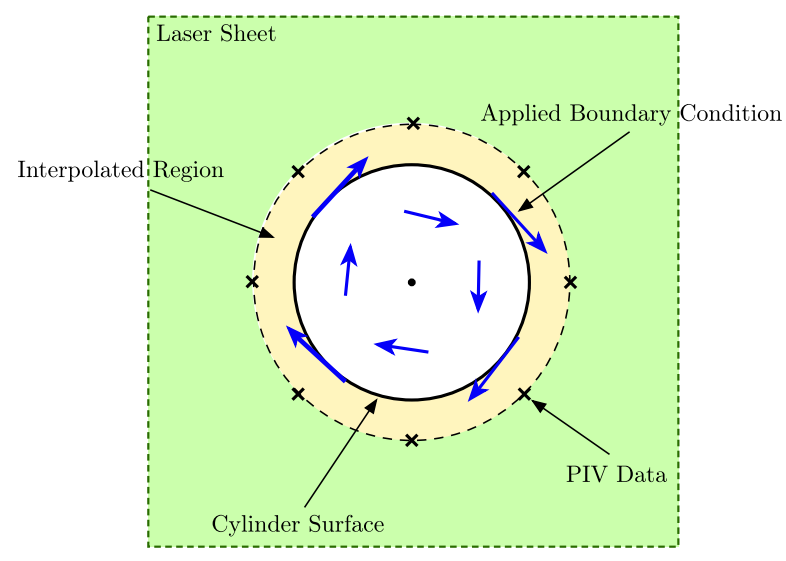

Fig. 8 Accounting for the missing PIV data by assigning the boundary condition to the cylinder.

Further to this, reflections of the laser sheet from the surface can lead to incomplete information close to the cylinder. Previous approaches [14-16] have shown promise in extracting force from PIV, but have not accounted for missing data near the surface. Graham et al. [10] accounted for the missing vorticity by proposing to determine the boundary 
layer vorticity from flow field measurements, thus greatly improving the accuracy. In the present case, the missing vorticity data is implicitly accounted for by applying the rotational and translational boundary condition of the cylinder kinematics to the cylinder surface. Interpolating between this specified cylinder surface condition and the known flow field recovers the missing vorticity data in the proximity of the cylinder. A schematic illustration of this is shown in figure 8. The missing vorticity in the real flow field could be located anywhere within the region of missing data. Therefore, whilst this method ensures that the correct magnitude of vorticity is included in the flow field, it does not guarantee the correct distribution in the wall normal direction. As to be expected, greater accuracy is achieved if the area of missing data becomes smaller.

To compute the forces, $F_{x}$ and $F_{y}$, the time rate of change of the impulse must be evaluated [13],

$$
F_{x}=-\rho \frac{d I_{x}}{d t}+\rho \sum_{j=1}^{N} \frac{d}{d t} \int_{A_{j}} u_{j} d A, \quad F_{y}=-\rho \frac{d I_{y}}{d t}+\rho \sum_{j=1}^{N} \frac{d}{d t} \int_{A_{j}} v_{j} d A
$$

where the impulse in the $x$ and $y$ direction is defined as

$$
I_{x}=\int_{A} y \omega d A, \quad I_{y}=-\int_{A} x \omega d A .
$$

$\rho$ is the density of the fluid, $u_{j}$ and $v_{j}$ are the translational velocities of any solid objects in the flow field of associated area $A_{j}$, for example the cylinder in the current investigation. The calculation of the impulse assumes a continuum of fluid everywhere in the domain, $A$. In the physical experiment, the region of the cylinder is occupied by the cylinder itself, rather than fluid. To account for this, the inertia of the displaced fluid must be subtracted from the total impulse to retrieve the acting forces; hence the appearance of the second term in equation 3

The mechanical circulation inherent to the solid body motion of the cylinder must be included in order for the impulse method to yield correct results [13]. Furthermore, it is necessary to consider this circulation to satisfy the conservation of circulation, as proposed by Kelvin [17]. Experimental proof of this is briefly reported in figure 9 It convincingly illustrates that excluding the circulation contained within the body of rotation leads to a non-zero total value of circulation. Only when the circulation within the body is considered, is the total circulation within the flow field zero and therefore conserved.

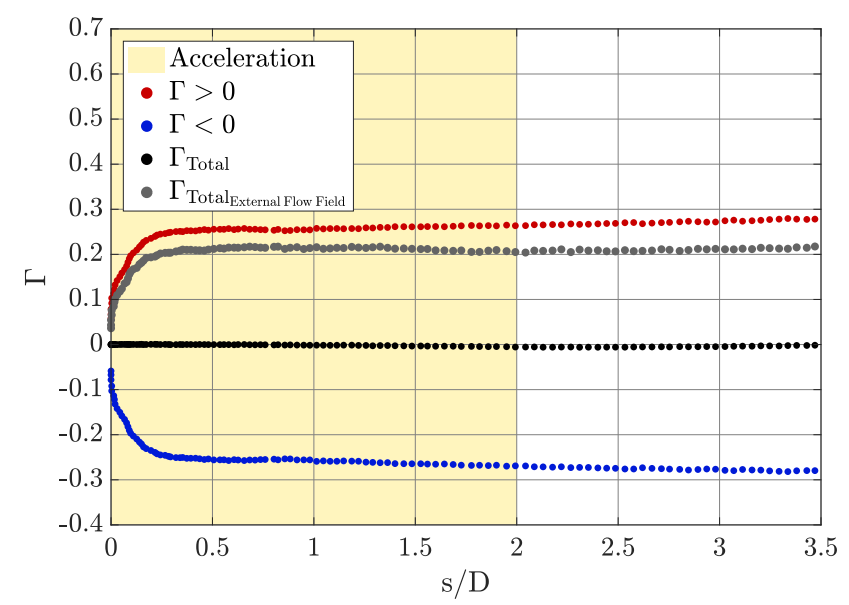

Fig. 9 Conservation of vorticity in the flow field. Case $2, R e=2 \times 10^{4}$ and $\alpha=3$.

\section{F. Vortex Sheet Computation}

The boundary layer vortex sheet, $\gamma_{b}$ and its components can be used to determine the respective circulatory and non-circulatory force components. To recover the individual components for a rotating body of finite thickness the approach outlined by Graham et. al [10] and Corkery \& Babinsky [9] is used and adapted. The boundary vortex sheet, 
$\gamma_{\mathrm{b}}$, is assumed to consist of three parts and defined as

$$
\gamma_{\mathrm{b}}=\gamma_{\mathrm{c}}+\gamma_{\mathrm{nc}}+\gamma_{\mathrm{rot}}
$$

Where $\gamma_{c}$ is the vortex sheet due to the shed vorticity and its respective mirror counterparts residing within the cylinder. $\gamma_{\text {rot }}$ is the vortex sheet due to the rotation of the cylinder. $\gamma_{\mathrm{nc}}$ is the non-circulatory vortex sheet, which is equivalent to the added mass vortex sheet, $\gamma_{\mathrm{am}}$, for a translating object. It enforces the no penetration condition along the surface due to only the kinematic motion of the body. Re-arranging equation 5 leads to

$$
\gamma_{\mathrm{am}}=\gamma_{\mathrm{b}}-\left(\gamma_{\mathrm{c}}+\gamma_{\mathrm{rot}}\right)
$$

$\gamma_{\text {rot }}$ is found by evaluating the vortex sheet strength due to the rotational motion of the cylinder in a quiescent fluid. It is therefore equivalent to the tangential velocity of the cylinder surface,

$$
\gamma_{\mathrm{rot}}=U_{\text {fluid }}-(-\Omega) a=0+U_{\theta}=U_{\theta} .
$$

$\gamma_{\mathrm{c}}$ is evaluated by finding the velocity imposed on the surface of the cylinder due to the shed circulation and the corresponding mirror counterparts. A schematic illustration of this is shown in figure 10a In a real viscous fluid, the physical mechanism to account for the mirror image of the shed circulation is the circulation residing in the boundary layer. Therefore, when calculating $\gamma_{c}$ from experimental data, the circulation contained within the boundary layer is disregarded. A visual representation of this is shown in figure $10 \mathrm{~b}$
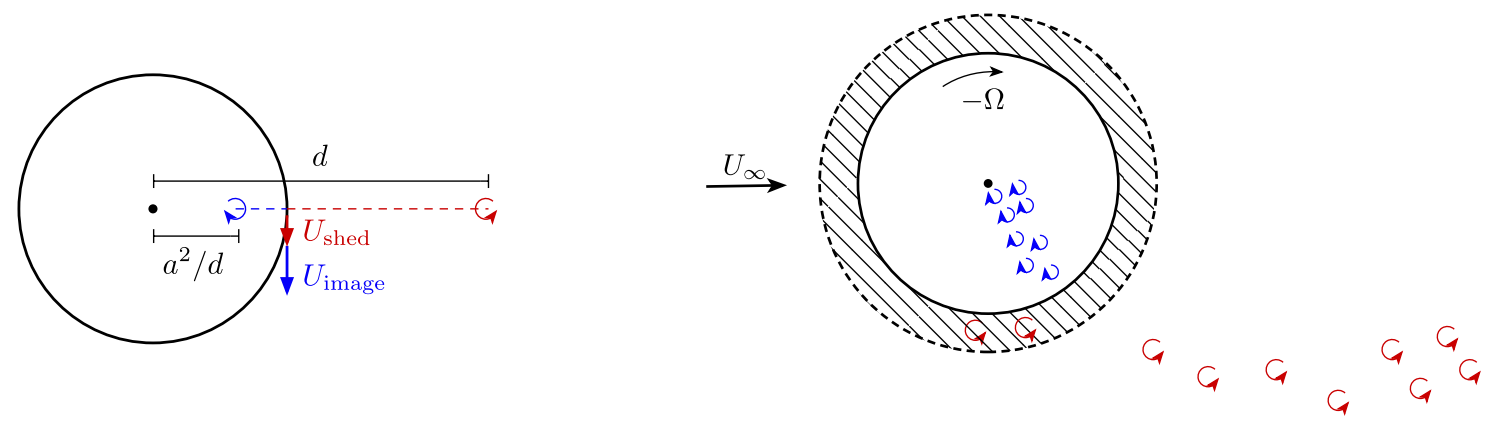

(a) Induced velocity due to an element of shed vorticity, a (b) Shed vorticity elements and mirrored counterparts. Vordistance $d$ away and its mirror image around a cylinder of ticity contained within the boundary layer (hashed region) is radius $a$. excluded.

Fig. 10 Determining $\gamma_{c}$ due to shed vorticity around a cylinder.

Mathematically, the velocities due to the elements of shed circulation are found by first defining the complex potential for $n$ vortices located in the flow field and their mirrored counterparts,

$$
F(z)=\sum_{j=1}^{n}-\frac{i \Gamma_{j}}{2 \pi}[\underbrace{\ln \left(z-d_{j} e^{i \varphi_{j}}\right)}_{\text {shed circulation }}-\underbrace{\ln \left(z-\frac{a^{2}}{d_{j}} e^{i \varphi_{j}}\right)}_{\text {mirror image }}]
$$

where $z=a e^{i \theta}$ indicates a position on the cylinder surface. $d$ is the distance between the circle centre and the element of circulation and $\varphi$ is the angle from the cylinder centre to the respective vortical element. Differentiating the complex potential with respect to $z$ yields the induced $u$ and $v$ velocity components,

$$
\frac{\mathrm{d} F}{\mathrm{~d} z}=u-i v=-\sum_{j=1}^{n} \frac{i \Gamma_{j}}{2 \pi}\left(\frac{1}{z-d_{j} e^{i \varphi_{j}}}-\frac{1}{z-\frac{a^{2}}{d_{j}} e^{i \varphi_{j}}}\right) .
$$


The local vortex sheet strength on the cylinder surface $\gamma_{c}$ is now the velocity difference between a stationary cylinder and the induced velocity, $U_{\text {induced }}$,

$$
\gamma_{c}=0-U_{\text {induced }}=-U_{\text {induced }}
$$

$\gamma_{\boldsymbol{b}}$ is determined by adapting the method proposed by Corkery \& Babinsky [9], thereafter referred to as the wedge method. The circumference of the cylinder is divided into a number of small wedges with an inner segment length $\delta s_{n}$, as illustrated in figure 11. The circulation for each wedge can be found by integrating the tangential velocity at the perimeter of the wedge according to $\delta \Gamma_{n}=\oint u d l$. When no velocity vector from the PIV data lies exactly on the wedge path, the PIV velocity field is linearly interpolated in the $x$ and $y$ direction to obtain the respective velocity along the wedge circumference. The velocity along the inner arc, situated on the surface, is set to the real mechanical velocity of the cylinder. This is obtained by tracking the translation velocity and rotation rate. Having obtained $\delta \Gamma_{n}$ for each segment, this can be divided by the segment length to obtain the vortex sheet strength for each element,

$$
\gamma_{n}=\frac{\delta \Gamma_{n}}{\delta s_{n}}
$$

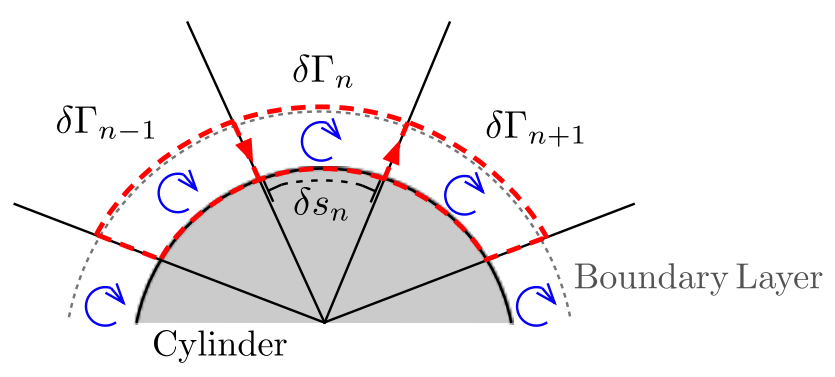

Fig. 11 Schematic illustration of the discretization of the flow field around the cylinder to obtain $\gamma_{b}$

\section{Results}

\section{A. Particle Image Velocimetry}

Prior to evaluating the vortex sheet components and the development of the boundary layer vortex sheet, PIV results are presented to provide an overview of the changing flow fields for the different kinematic cases.

Flow around a rotating cylinder is classified into two main categories. Alternate vortex shedding from either side of the cylinder occurs for values of $\alpha$ below approximately 1.9 [18, 19]. When $\alpha$ exceeds this value, vortex shedding is suppressed and only one initial starting vortex is shed [18]. A further regime exists when $4.34<\alpha<4.70$ [19] but this can be disregarded for the present study.

\section{Case 1 - Surging Cylinder}

The vorticity contours for Case 1, translation only, from $s / D=0.5$ to $s / D=4.5$ are presented in figure 12 . Vorticity of approximately equal magnitude is first generated on either side of the cylinder as it begins to move. With increasing distance, the vorticity rolls up into two distinct primary vortices. As the vortices become stronger, their induced velocity causes vorticity of the opposite sign to be generated on the cylinder surface. This can be seen in figures 12d through $12 \mathrm{f}$ The positive and negative separation points, $\left.\overline{\gamma_{b}}\right|_{+ \text {ve sep. loc. }}$ and $\left.\overline{\gamma_{b}}\right|_{- \text {ve sep. loc. }}$, are found by assessing the variation of $\gamma_{b}$ normalised by $U_{\infty}, \overline{\gamma_{b}}$, and are also indicated in figure 12 . More detail on this will be given in section IV.D

It is important to note, that the thickness of the region occupied by the positive and negative vorticity may be exaggerated in the figure. This is because interpolating over the region of missing PIV data falsely causes vorticity to visually be represented to occupy the entire area. However, whilst the size of the region is incorrectly displayed, the magnitude of the circulation found within is still correct. 


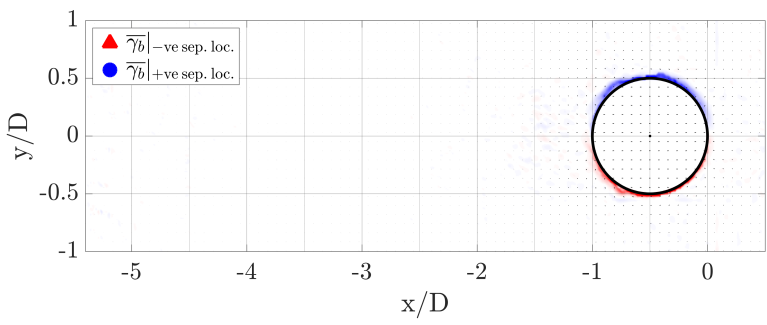

(a) $s / D=0.5$.

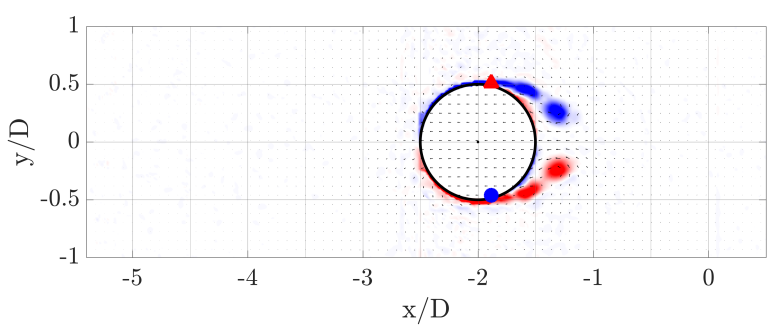

(c) $s / D=2.0$.

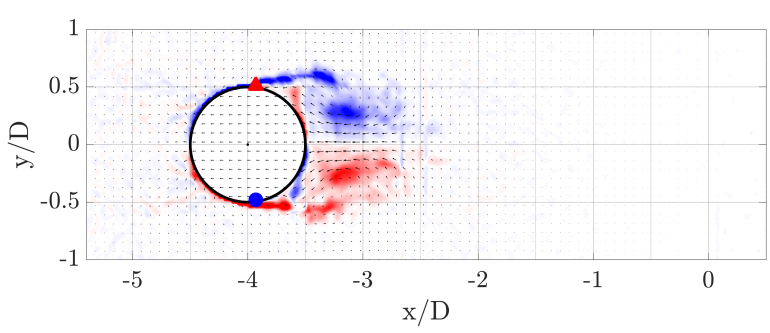

(e) $s / D=4.0$.

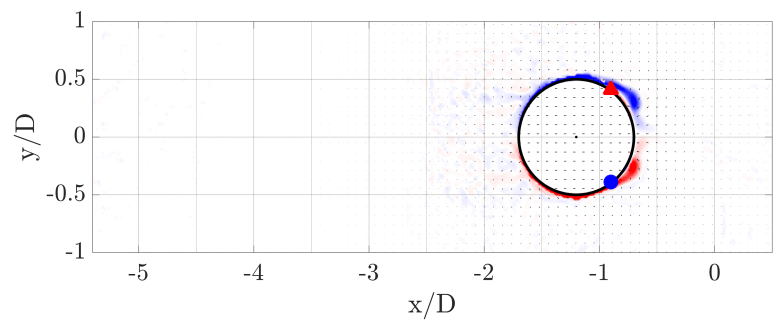

(b) $s / D=1.2$.

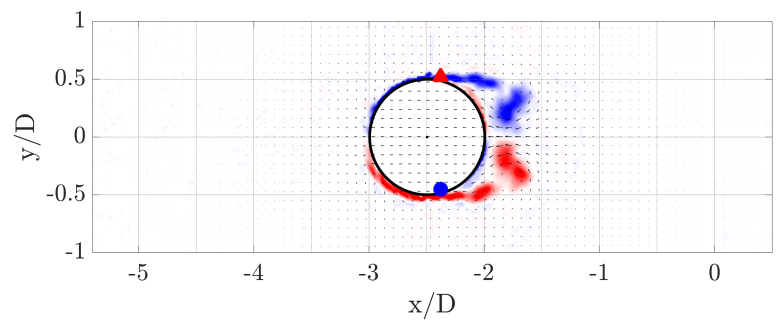

(d) $s / D=2.5$.

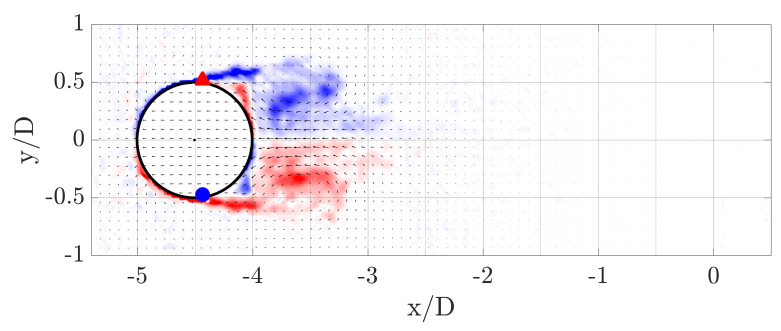

(f) $s / D=4.5$.

Fig. 12 Normalised vorticity contours as the cylinder begins to translate. Case $1, R e=2 \times 10^{4}$.

\section{Case 2 - Translation and Rotation}

Introducing rotation, the vorticity contours for Case 2 when $\alpha=1, R e=2 \times 10^{4}$ and $s_{a c c}=2$ are presented in figure 13 for $s / D=0.5$ to $s / D=4.75$. The vorticity within the cylinder is also depicted and stems from the associated solid body rotation. Initially, positive vorticity forms around most of the cylinder as the relative velocity due to rotation dominates. As it translates, the positive vorticity begins to roll up into a vortex and sheds from the bottom edge. Simultaneously, negative vorticity grows on the upper half and sheds from the upper surface, as seen in figure $13 \mathrm{f}$. This indicates that alternate vortex shedding is taking place and measurements during the steady state confirm this. It consequently matches results in literature reporting alternate vortex shedding below $\alpha=1.91$ [19]. 


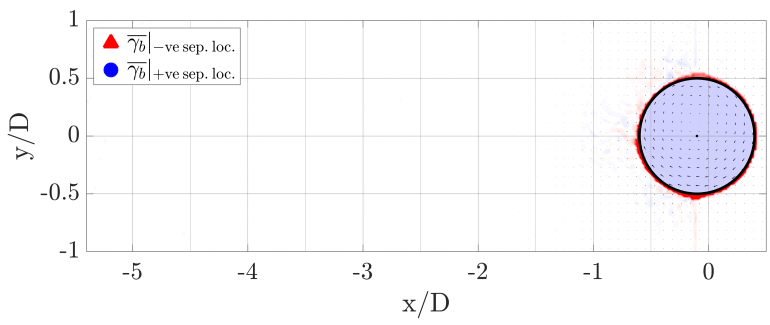

(a) $s / D=0.1$.

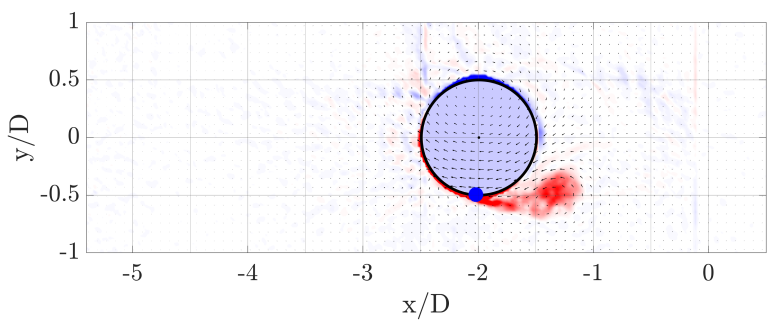

(c) $s / D=2.0$.

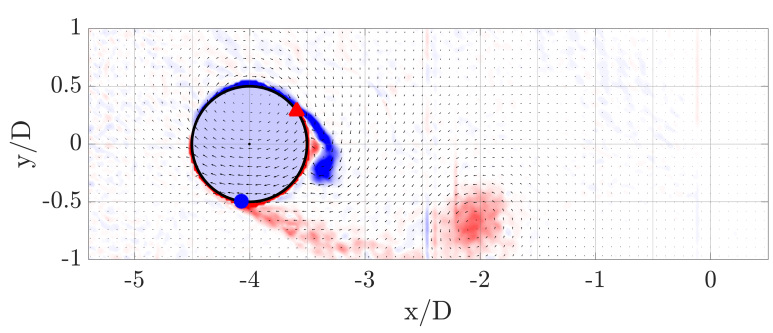

(e) $s / D=4.0$.

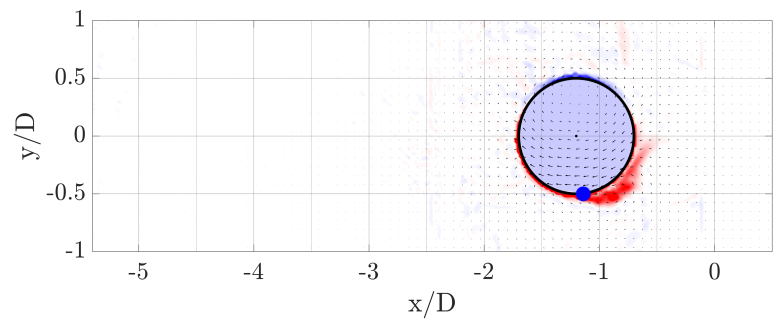

(b) $s / D=1.2$.

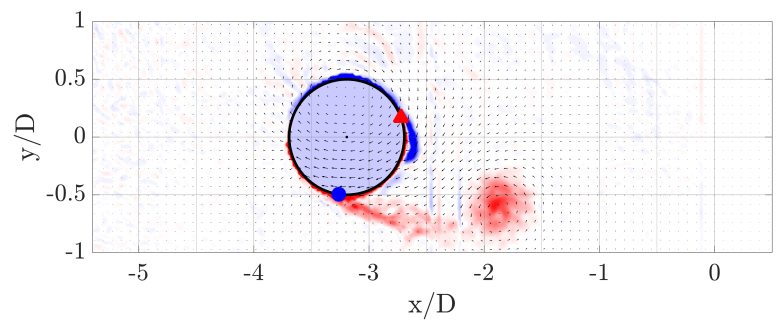

(d) $s / D=3.2$.

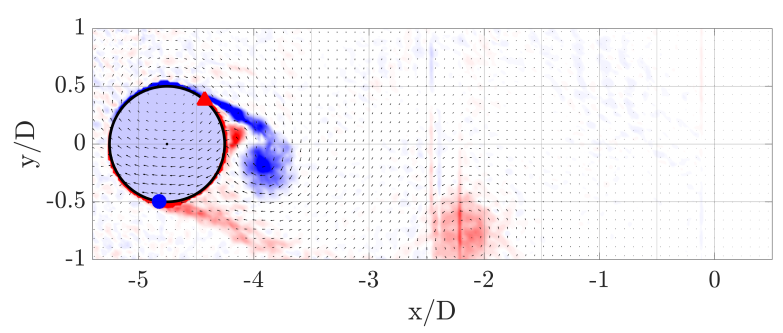

(f) $s / D=4.75$.

Fig. 13 Normalised vorticity contours as the cylinder begins to translate and rotate. Case $2, R e=2 \times 10^{4}$ and $\alpha=1$.

Increasing the rotation rate such that $\alpha=3$ leads to the vorticity contours shown in figure 14 for $s / D=0.1$ to $s / D=4$. Initially, as the cylinder reaches its terminal angular velocity within $s / D=0.3$, positive vorticity is generated in the flow field around the cylinder. This is however, not yet shed. As the cylinder continues to accelerate, the expected single starting vortex is formed and sheds into the flow field as can be seen in figures $14 \mathrm{c}$ through $14 \mathrm{f}$ The absence of an alternating vortex shedding pattern confirms the results in literature for the regime above $\alpha=1.91$ [18]. 


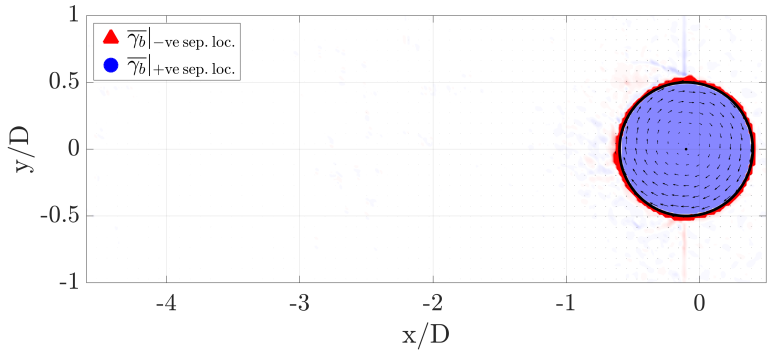

(a) $s / D=0.1$.

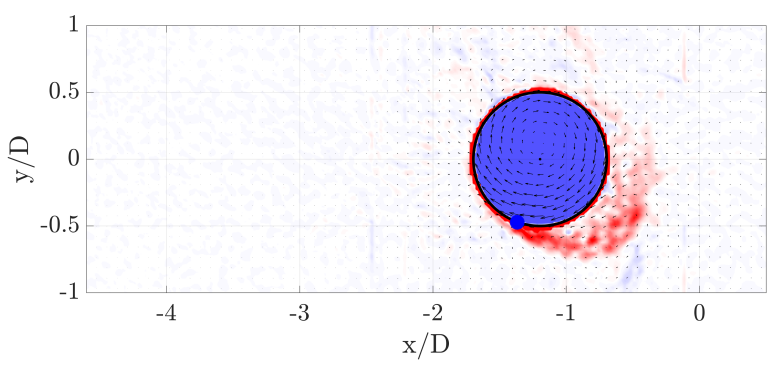

(c) $s / D=1.2$.

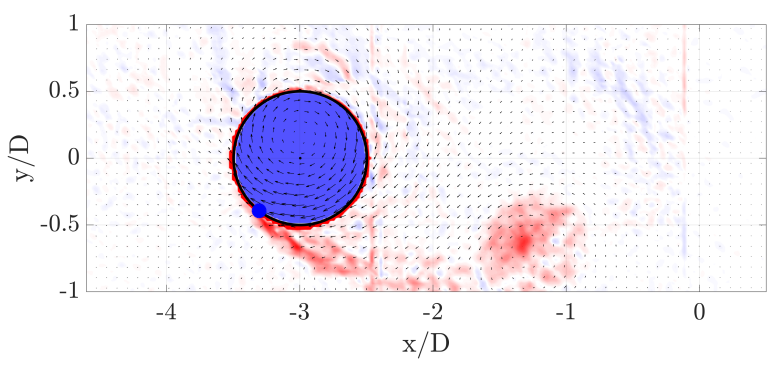

(e) $s / D=3.0$.

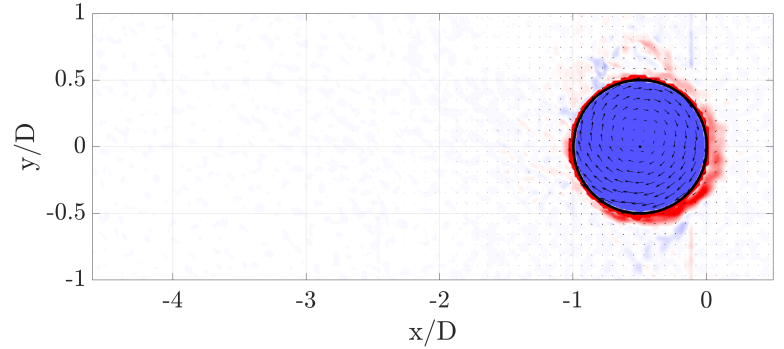

(b) $s / D=0.5$.

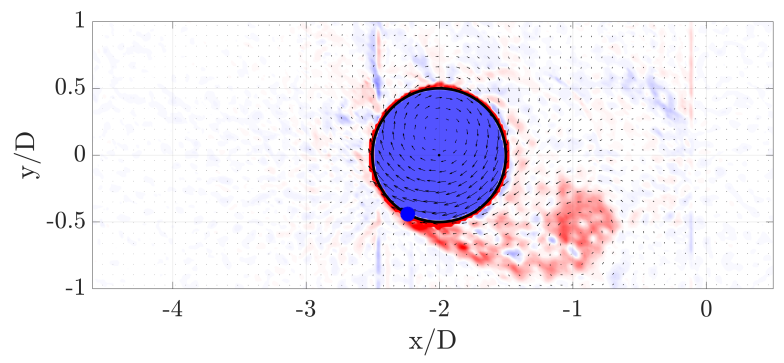

(d) $s / D=2.0$.

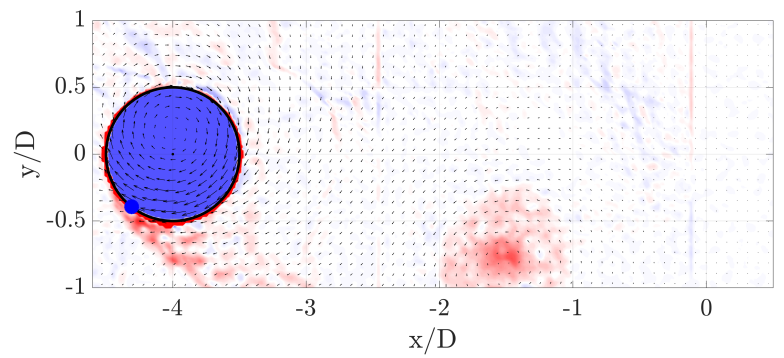

(f) $s / D=4.0$.

Fig. 14 Normalised vorticity contours as the cylinder begins translation and rotation Case $2, R e=2 \times 10^{4}$ and $\alpha=3$.

\section{B. Vortex Sheets}

\section{Vortex Sheet Decomposition}

To investigate the unsteady force generation the surface vortex sheet is split up into its individual components according to the methodology presented in section III.F. Here, the aim is to recover the non-circulatory vortex sheet. The result of such a decomposition for a purely translating cylinder is shown in figure $15 \mathrm{a}$, where $\gamma_{b}$ is non-dimensionalized using the instantaneous velocity, $U$. The expected added mass vortex sheet for a translating circular cylinder is included for comparison, $\gamma_{\mathrm{am}_{\text {Theory }}}$.

As $\gamma_{\mathrm{nc}}$ is only a function of instantaneous translation velocity, it is possible to reduce the noise in the final vortex sheet distribution. Rather than averaging $\gamma_{\mathrm{nc}}$ at one particular instance in time over the individual runs, an average of $\gamma_{\mathrm{nc}} / U$ can be taken over each run as well as over each PIV frame whilst the cylinder is translating. Since $\gamma_{\mathrm{b}}$ and $\gamma_{\mathrm{c}}$ are not purely a function of $U$, the corresponding distributions in figure 15 a are not entirely correct, however, they are included for reference to illustrate the difference in the vortex sheet components between Case 1 and Case 2 . The vortex sheet components for Case 2 when $\alpha=3$ are presented in figure $15 \mathrm{~b}$. Comparing the theoretical added mass and the experimental non-circulatory vortex sheet clearly shows that it is possible to recover $\gamma_{\mathrm{nc}}$ from PIV data. A similar result was previously shown by Corkery \& Babinsky [9] for a translating thin flat plate. The data presented here confirms that this approach can be extended to objects of finite thickness. 


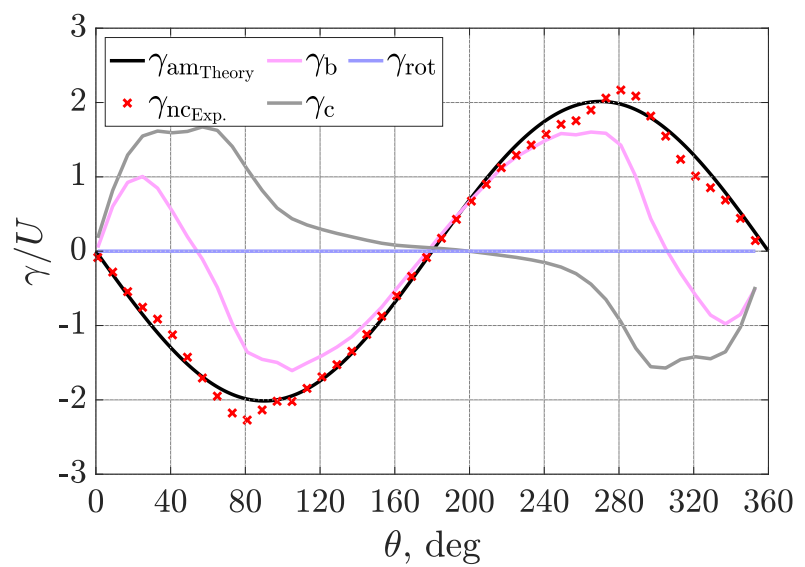

(a) Case 1.

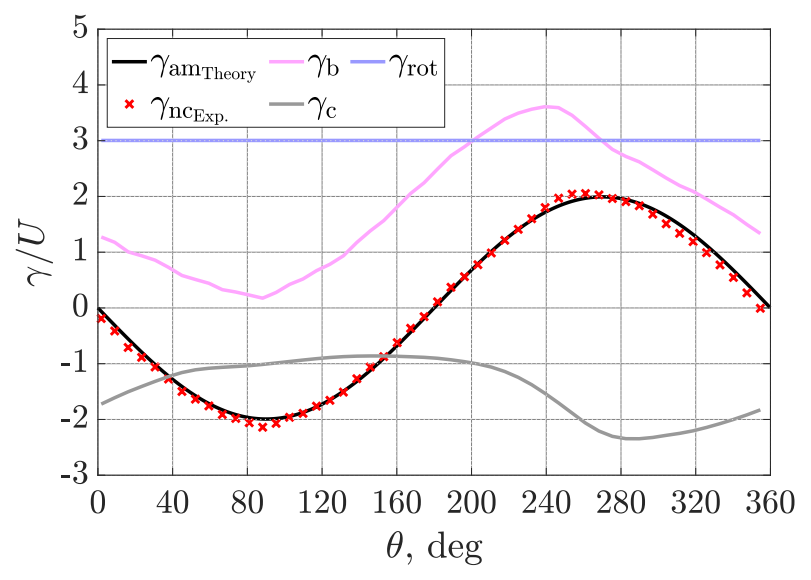

(b) Case 2, $\alpha=3$.

Fig. $15 \gamma / U$ averaged over individual runs and all time instances. Compared to $\gamma_{\text {am }}$ Theory obtained from potential flow. $R e=2 \times 10^{4}$.

The implication of being able to measure $\gamma_{\mathrm{nc}}$ is that it allows the forces computed from PIV measurements to be split up into their circulatory and non-circulatory counterparts. This will be shown in the following section. It does not only help the creation of low order models but it gives a researcher or engineer an intuitive indication of where the generated forces stem from. On a theoretical level, it demonstrates the link between the added mass force and vorticity for an accelerating body and confirms that these two cannot be viewed to be independent.

\section{Impulse Method - Applied to Data}

The successful decomposition of the surface vortex sheet can now be used to demonstrate that it is possible to recover the force components acting on the cylinder from PIV measurements. The comparison between the forces calculated from PIV and the force balance measurements for Case 2, when $\alpha=1$ as well as for $\alpha=3$ when $\operatorname{Re}=2 \times 10^{4}$ and $s_{a c c}=2$ are presented in figures 16 and 17 Generally $C_{L}, C_{D}$ and $C_{D_{a m}}$ obtained from PIV measurements match well to the force balance data for both rotation rates, as long as all flow field vorticity is within the field of view and the flow is two-dimensional. With increasing translational distance, the wake will tend to become more three-dimensional, hence introducing errors into the measured vorticity of the shed vortex, and subsequently causing the observed deviation.

It can be seen in both figure 16 and figure 17 that assigning the boundary condition and interpolating over the missing flow field greatly improves the force approximation. This incorporates the vorticity close to the cylinder surface, which is otherwise hidden in the missing vorticity data. The effect is most pronounced whenever changes in the vorticity generation at the surface occur too quickly for the vorticity to diffuse away from the cylinder and into the measurable flow field.

The theoretical added mass force coefficient included in figures 16 and 17 is calculated according to

$$
F_{a m}=\rho_{w} \pi a^{2} l \dot{U}
$$

where $\rho_{w}$ is the density of the water and $l$ is the cylinder span. The added mass contribution obtained from the PIV data is computed by evaluating the time rate of change of the impulse of the added mass vortex sheet,

$$
F_{x}=\frac{d I_{x}}{d t}=-\frac{d}{d t} \rho \oint y \gamma_{a m} d l+\rho \frac{d}{d t} \int_{A_{c}} u d A .
$$

$u$ is the velocity of the cylinder and $A_{c}$ the respective area. The second term in equation 13 is required to remove the inertia of the fluid displaced by the cylinder from the total impulse, as the cylinder has a finite thickness. The close match between the theoretical and the through PIV measurements experimentally obtained added mass, not only confirms that 


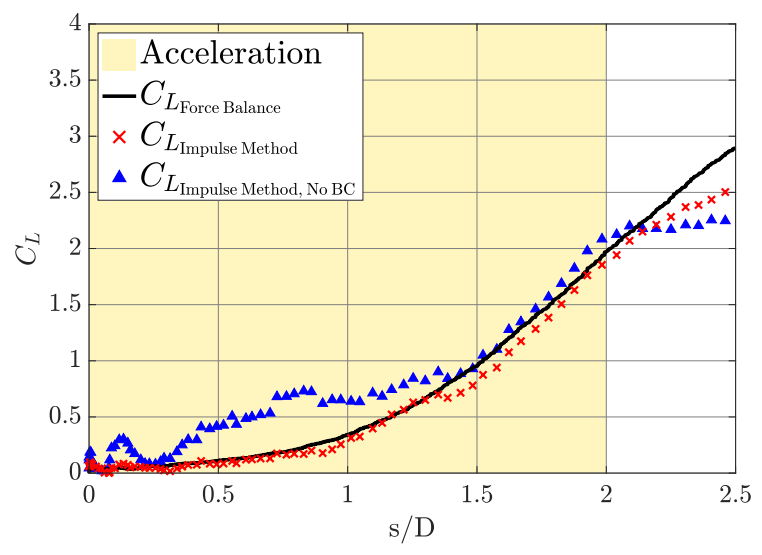

(a) $C_{L}$ evolution.

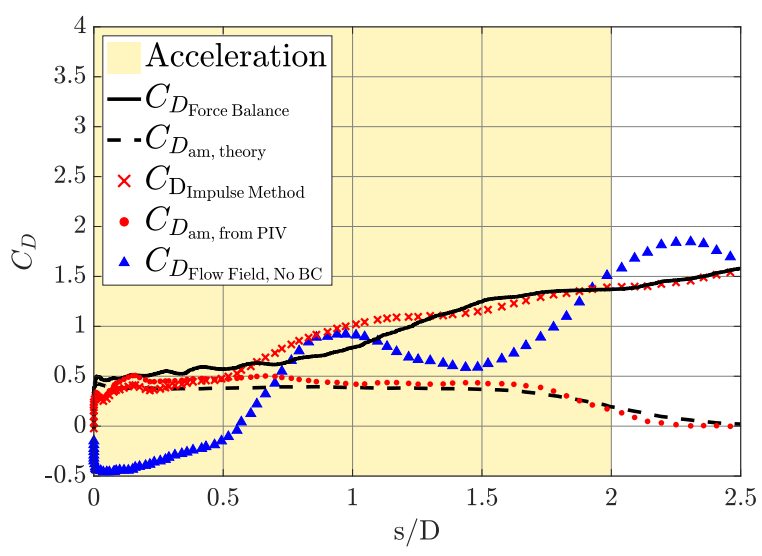

(b) $C_{D}$ evolution.

Fig. $16 C_{L}$ and $C_{D}$ obtained from PIV and force measurements. $\alpha=1$.

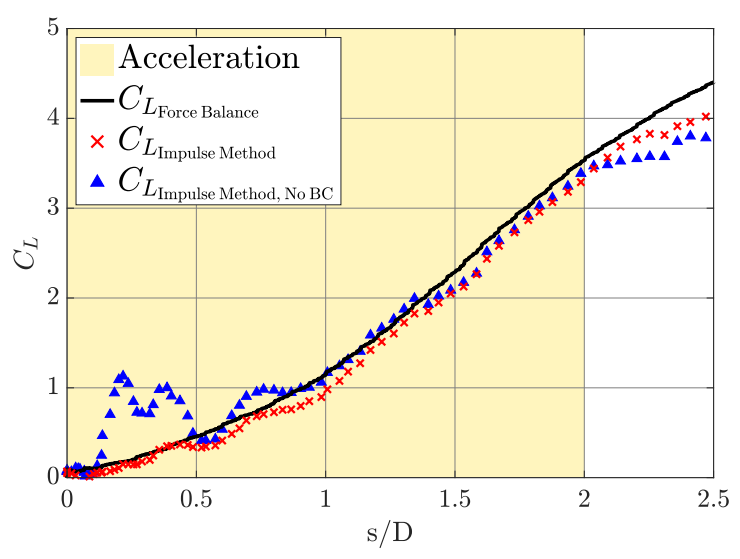

(a) $C_{L}$ evolution.

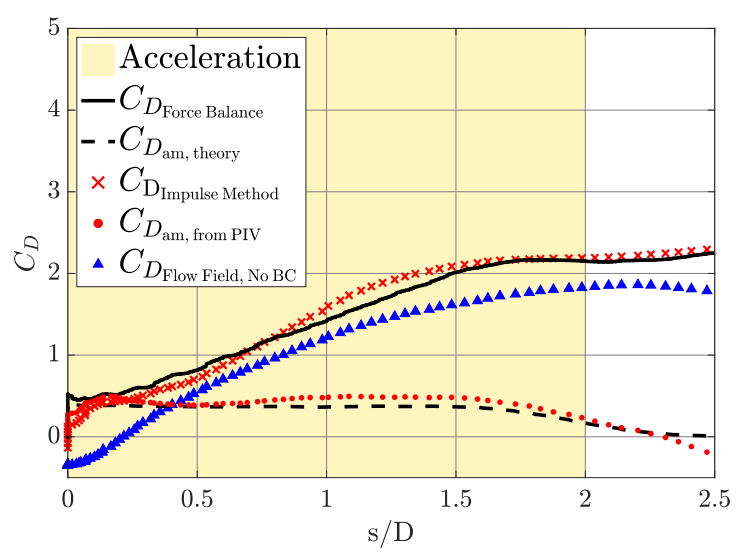

(b) $C_{D}$ evolution.

Fig. $17 C_{L}$ and $C_{D}$ obtained from PIV and force measurements. $\alpha=3$.

an added mass vortex sheet can be measured in the flow field, but that a good approximation of the corresponding force contribution can also be obtained. This has now been shown to be possible regardless of whether the body is of infinite or finite thickness or has a non-zero rotational component.

\section{Evolution of $\gamma_{b}$}

The evolution of $\gamma_{b}$ can be tracked as the cylinder begins to translate and rotate. During the initial phase, distinguishing between $\gamma_{b}$ and $\gamma_{c}$ is difficult because of limited resolution directly around the cylinder. While the separation develops, some of the shed vorticity is erroneously included in the determination of $\gamma_{b}$ which leads to an incorrect distribution of $\gamma_{b}$. Once the separating shear layer is defined, for $s / D \geqslant 1$, a clear measurement of $\gamma_{b}$ is possible.

Non-dimensionalising by $U_{\infty}$ and plotting the resulting distribution $\overline{\gamma_{b}}$ for one instance in time for a surging cylinder yields the distribution shown in figure $18 \mathrm{a}$. The theoretical vortex sheet if separation is ignored, $\bar{\gamma}_{\text {theo.inst. }}$ is also included. When $80^{\circ}<\theta<280^{\circ} \overline{\gamma_{b}}$ roughly follows a sinusoidal shape, albeit with a lower amplitude than $\bar{\gamma}_{\text {theo.inst. }}$. Here the flow is attached and $\overline{\gamma_{b}}$ is dominated by $\gamma_{n c}$. The distinct sinusoidal shape comes from the contribution of $\gamma_{n c}$, which varies with $-2 U \sin \theta$. Regions of $\overline{\gamma_{b}}$ opposite in sign to the shed vorticity are found below $\theta \approx 50^{\circ}$ and above $\theta \approx 310^{\circ}$. This is because of the induced flow by the separating vortices. 
At $\theta \approx 83^{\circ}$ and $\theta \approx 277^{\circ} \overline{\gamma_{b}}$ experiences a sudden change in gradient and rises or drops to $\overline{\gamma_{b}} \approx 0$. The points are indicated on the distribution of $\overline{\gamma_{b}}$ in figure $18 \mathrm{a}$ and also plotted at the corresponding locations on the vorticity contour plot in figure $18 \mathrm{~b}$. The sudden change in $\overline{\gamma_{b}}$ coincides with where the shear layers leave the cylinder surface in figure $18 \mathrm{~b}$ and consequently denote the separation points. Vorticity is ejected from the cylinder surface and rolls up to form a vortex and hence no longer contributes to $\overline{\gamma_{b}}$.

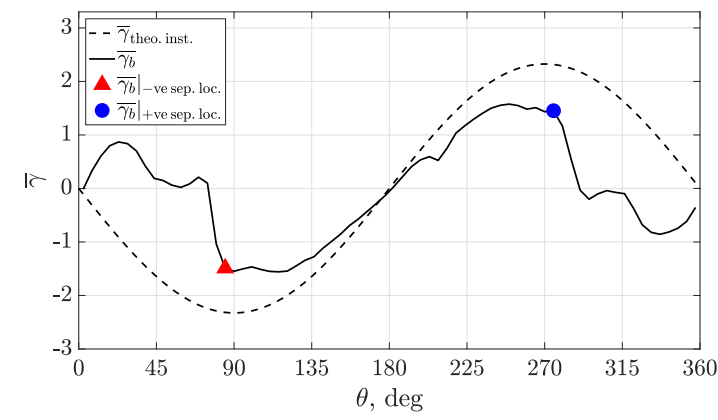

(a) $\overline{\gamma_{b}}$ and vortex sheet assuming no shed vorticity.

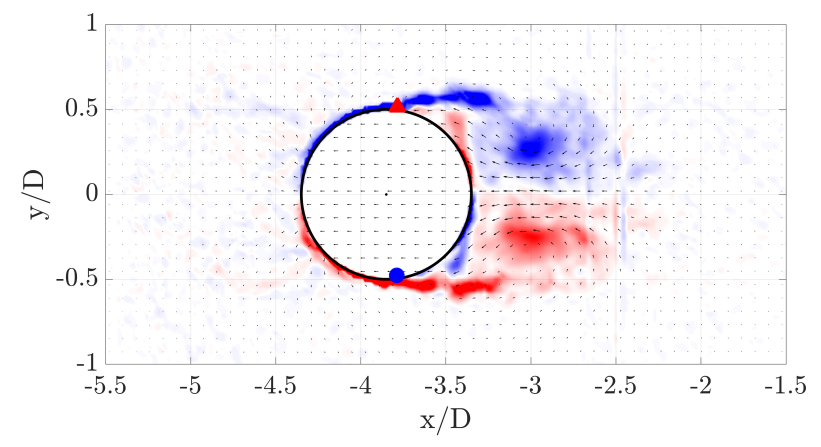

(b) Normalised vorticity contours.

Fig. $18 \overline{\gamma_{b}}$ at $s / D=3.85$. Separation points found from the drop in $\overline{\gamma_{b}}$ are also indicated. Case $1, \operatorname{Re}=2 \times 10^{4}$, $s_{\text {acc }}=3$.

To remove the effect of varying instantaneous translational and rotational velocities $\gamma_{b}$ can be non-dimensionalised by the maximum instantaneous cylinder surface velocity $U+U_{\theta}$ such that

$$
\widetilde{\gamma_{b}}=\frac{\gamma_{b}}{U+U_{\theta}}
$$

The development of $\widetilde{\gamma_{b}}$ for the surging cylinder is presented in figure 19 for $s / D=1$ to $s / D=4.5$. For this small translation distance the vortex sheet is symmetrical around the top and bottom half of the cylinder. Therefore figure 19 only shows the bottom half, $180^{\circ} \leqslant \theta \leqslant 360^{\circ}$.

As the cylinder begins to translate, the location of the drop in $\widetilde{\gamma_{b}}$ and consequently the separation point is found to move upstream. $\widetilde{\gamma_{b}}$ at the separation point will be termed ${\widetilde{\gamma_{b}}}_{\text {crit }}$ and the corresponding angle $\theta_{\text {crit }}$. Interestingly, ${\widetilde{\gamma_{b}}}_{c r i t}$ remains approximately constant, even though, the separation location and hence the flow field is changing as seen in figure 12. Here the separation points, identified by the change in slope of the surface vortex sheet, are also indicated.

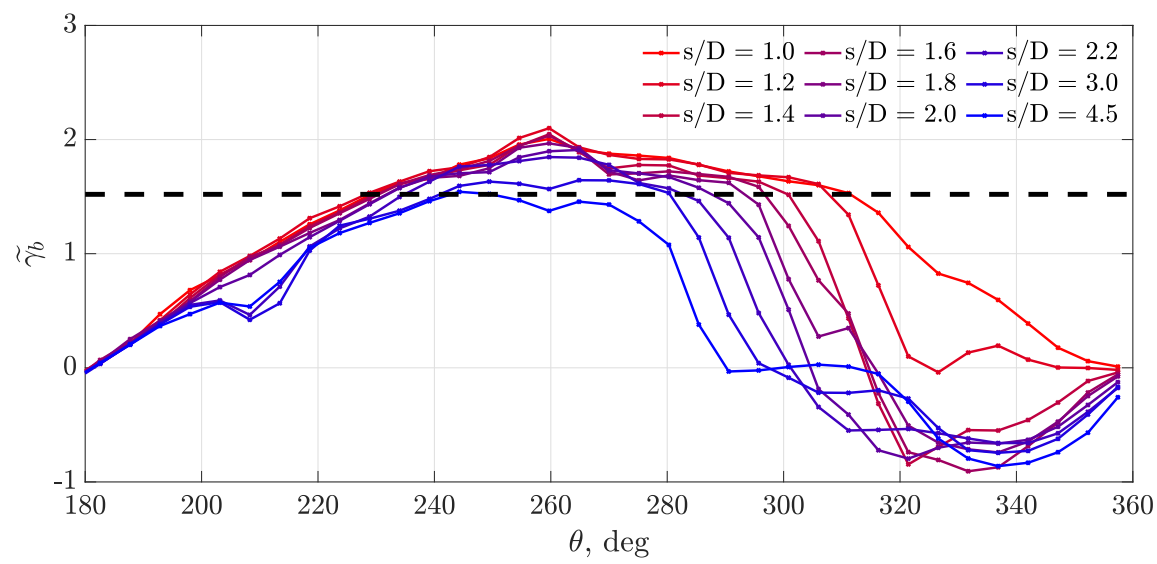

Fig. 19 Evolution of $\widetilde{\gamma_{b}}$. The horizontal dashed line indicates the approximate value of ${\widetilde{\gamma_{b}}}_{\text {crit }}$ at the points of separation. Case 1, $R e=2 \times 10^{4}, s_{a c c}=3$. 
The development of the surface vortex sheet can equally be measured and recorded for Case 2, when the cylinder is rotating and translating. To begin, $\overline{\gamma_{b}}$ when a vortex from both sides of the cylinder has been shed together with $\bar{\gamma}_{\text {theo.inst. }}$ is presented in figure 20a. The cylinder is at $s / D=4.75$ and the normalised vorticity contours are shown in figure $20 \mathrm{~b}$

Similar to before, the left side of the cylinder, $49^{\circ}<\theta<270^{\circ}$, features the characteristic sinusoidal distribution due to the $\gamma_{n c}$. The flow remains fully attached. At $\theta=49^{\circ}$ and $\theta=270^{\circ} \overline{\gamma_{b}}$ features a sudden change in gradient with the respective locations indicated in both figure $20 \mathrm{a}$ and $20 \mathrm{~b}$. Once more, it is observed that the points coincide with where the shear layer leaves the surface of the cylinder and thus with the separation points.

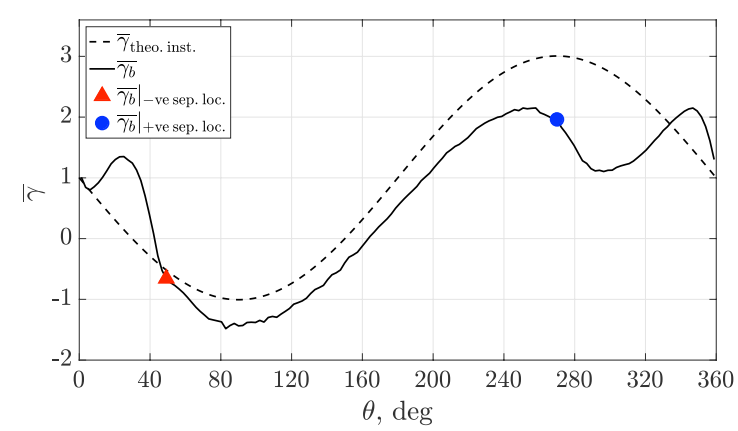

(a) $\overline{\gamma_{b}}$ and vortex sheet assuming no shed vorticity.

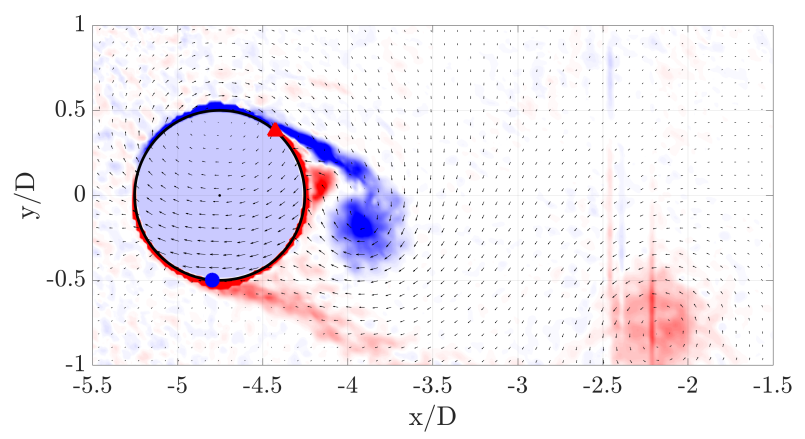

(b) Normalised vorticity contours.

Fig. $20 \overline{\gamma_{b}}$ at $s / D=4.75$ Separation points found from the drop in $\overline{\gamma_{b}}$ are also indicated. Case 2, $\alpha=1$, $R e=2 \times 10^{4}, s_{a c c}=2$.

The evolution of $\widetilde{\gamma_{b}}$ for $1 \leqslant s / D \leqslant 4.5$, once the vorticity associated to $\gamma_{b}$ and $\gamma_{c}$ can be clearly identified, is shown in figure 21. A number of interesting features can be identified:

- The distribution of $\widetilde{\gamma_{b}}$ on the upstream moving half of the cylinder collapses leading up to the separation point when $1 \leqslant s / D \leqslant 2$, whilst at the same time, $\widetilde{\gamma_{b}}$ on the downstream moving wall is consistently decreasing.

- During the initial vortex formation phase, when $1 \leqslant s / D \leqslant 2, \gamma_{b_{c r i t}}$ at the separation point remains approximately constant even with small variations of the separation point.

- Once the vortex begins to detach, $s / D>2,{\widetilde{\gamma_{b}}}_{c r i t}$ drops to a new, lower constant value. When a second vortex is shed from the upstream moving wall, the initial high ${\widetilde{\gamma_{b}}}_{c r i t}$ is again reached during the formation process. The corresponding $\widetilde{\gamma_{b}}$ distribution is omitted from figure 21 for clarity.

- ${\widetilde{\gamma_{b}}}_{c r i t}$ at the separation location on the downstream moving wall varies minimally even for strongly changing separation locations $18^{\circ} \leq \theta_{\text {crit }} \leq 43^{\circ}$. This can be seen in figure 21 between $s / D \approx 3.7$ and $s / D \approx 4.5$. The location of the separation points, as found from the change in gradient of the surface vortex sheet is also indicated in figure 13 


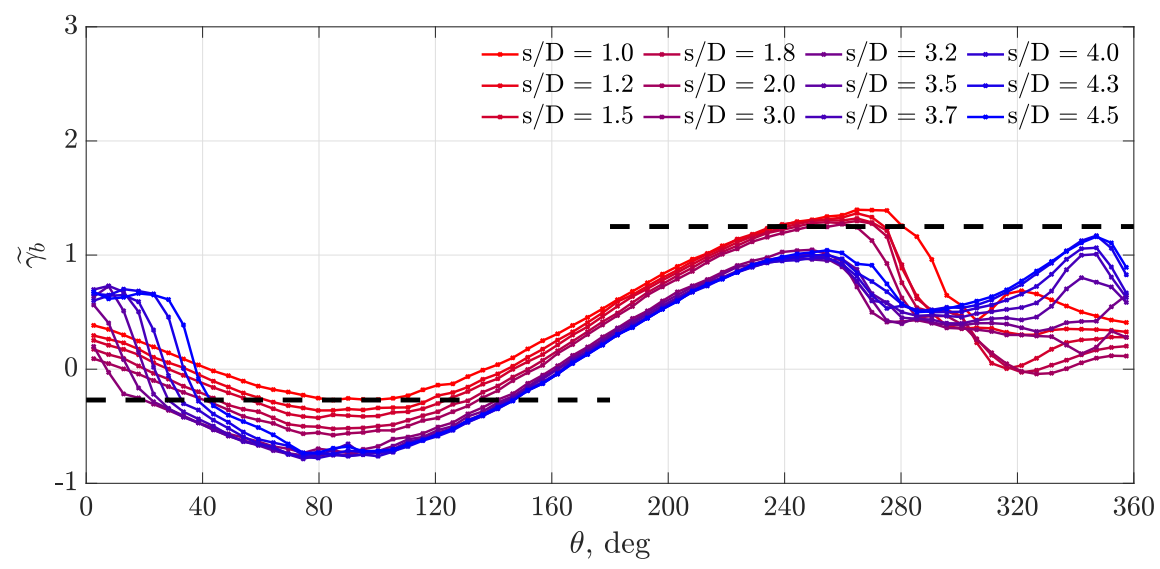

Fig. 21 Evolution of $\widetilde{\gamma_{b}}$. The horizontal dashed lines indicates the approximate value of ${\widetilde{\gamma_{b}}}_{\text {crit }}$ at the points of separation. Case 2, $\alpha=1, \operatorname{Re}=2 \times 10^{4}, s_{a c c}=2$.

The described behaviour of $\widetilde{\gamma_{b}}$ at the separation points show distinct similarities to a trend reported by Melius et al. who studied the unsteady separation point on a pitching aerofoil [20]. The authors suggested that there is a specific value of surface vorticity that could be sustained before separation occurred. The measurements of surface vorticity were however, confined to a single point. In the present study, the surface vortex sheet can be fully recovered through direct measurements of the complete boundary layer.

The distribution and development of $\widetilde{\gamma_{b}}$ on the downstream moving wall also demonstrates that the magnitude of $\widetilde{\gamma_{b}}{ }_{c i t}$ can be exceeded without separation occurring. The minimum is reached at $\theta=90^{\circ}$ after which $\widetilde{\gamma_{b}}$ increases smoothly, following a sin curve, until it reaches the separation point. This indicates that separation must not occur at the maximum absolute value of the surface vortex sheet.

\section{Conclusion}

A surging and rotating circular cylinder is used to demonstrate that the unsteady force experienced during acceleration can be determined from the boundary layer vortex sheet and its respective components. The non-circulatory vortex sheet component, for an accelerating body, leads to the recovery of the added mass force component, whilst the evolution of the vortex sheet itself provides some information about separation and thus, the circulatory force component. The key results presented in this investigation are:

- The surface vortex sheet of a rotating body of finite thickness in an experimental setting can be decomposed into a non-circulatory component, caused by added mass effects, a component due to the rotation of the body and a component due to shed vorticity. This methodology allows the assessment of the contribution of individual components to the evolution of the surface vortex sheet for a body of finite thickness.

- The lift and drag forces can be recovered from PIV data and are found to match well with force balance measurements. The correct boundary condition must, however, be assigned to the cylinder surface to account for incomplete PIV data close to the cylinder.

- The added mass force calculated from the impulse of the experimentally obtained added mass vortex sheet correlates well with the theoretical value.

- The strength of the surface vortex sheet, when removing the effect of varying free stream velocity and spin rate, remains approximately constant at the point of separation. Crucially this appears to be the case, even as the separation point moves upstream. Observations of this phenomena apply to both separation on the upstream and downstream moving wall. 


\section{Acknowledgments}

The author would like to acknowledge the Engineering and Physical Science Research Council (EPSRC) for providing financial support.

\section{References}

[1] Watkins, S., Milbank, J., Loxton, B. J., and Melbourne, W. H., “Atmospheric Winds and Their Implications for Microair Vehicles," AIAA Journal, Vol. 44, No. 11, 2006, pp. 2591-2600.

[2] Watkins, S., Thompson, M., Loxton, B., and Abdulrahim, M., "On Low Altitude Flight Through The Atmospheric Boundary Layer," International Journal of Micro Air Vehicles, Vol. 2, No. 2, 2010, pp. 55-68.

[3] White, C., Lim, E. W., Watkins, S., Mohamed, A., and Thompson, M., “A Feasibility Study of Micro Air Vehicles Soaring Tall Buildings," Journal of Wind Engineering and Industrial Aerodynamics, Vol. 103, 2012, pp. 41-49.

[4] Pitt Ford, C. W., and Babinsky, H., "Lift and the Leading-Edge Vortex," Journal of Fluid Mechanics, Vol. 720, 2013, pp. 280-313.

[5] Stevens, P. R. R. J., and Babinsky, H., "Experiments to Investigate Lift Production Mechanisms on Pitching Flat Plates," Experiments in Fluids, Vol. 58, No. 1, 2017, pp. 1-17.

[6] Babinsky, H., and Stevens, P. R. R. J., "Low Order Modelling of Lift Forces for Unsteady Pitching and Surging Wings ." AIAA, 2016, pp. 1-12.

[7] Corkery, S. J., Stevens, P. R. R. J., and Babinsky, H., "Low Reynolds Number Surge Response of a Flat Plate Wing at 90 Degrees Incidence,” 55th AIAA Aerospace Sciences Meeting, 2017, pp. 1-16.

[8] Eldredge, J. D., "A Reconciliation of Viscous and Inviscid Approaches to Computing Locomotion of Deforming Bodies," Experimental Mechanics, Vol. 50, No. 9, 2010, pp. 1349-1353.

[9] Corkery, S. J., and Babinsky, H., "Force Production Mechanisms for a Flat Plate Wing at Low Reynolds Numbers," 2018 AIAA Aerospace Sciences Meeting, AIAA SciTech Forum, American Institute of Aeronautics and Astronautics, 2018.

[10] Graham, W. R., Pitt Ford, C. W., and Babinsky, H., “An Impulse-Based Approach to Estimating Forces in Unsteady Flow," Journal of Fluid Mechanics, Vol. 815, 2017, pp. 60-76.

[11] Westerweel, J., "Fundamentals of Digital Particle Image Velocimetry,” Meas. Sci. Technol, Vol. 8, 1997, pp. 1379-1392.

[12] Westerweel, J., "Efficient Detection of Spurous vectors in Particle Image Velocimetry Data," Experiments in Fluids, Vol. 16, 1994, pp. 236-247.

[13] Wu, J. C., "Theory for Aerodynamic Force and Moment in Viscous Flows," AIAA Journal, Vol. 19, No. 4, 1981 , pp. $432-441$.

[14] Lin, J. C., and Rockwell, D., "Force Identification by Vorticity Fields:Techniques Based on Flow Imaging," Journal of Fluids and Structures, Vol. 10, No. 6, 1996, pp. 663-668.

[15] Poelma, C., Dickson, W. B., and Dickinson, M. H., "Time-Resolved Reconstruction of the Full Velocity Field Around a Dynamically-Scaled Flapping Wing," Experiments in Fluids, Vol. 41, 2006, pp. 213-225.

[16] Devoria, A. C., and Ringuette, M. J., "On the Flow Generated on the Leeward Face of a Rotating Flat Plate," Experiments in Fluids, Vol. 54, No. 4, 2013.

[17] Kelvin, L., “On Vortex Motion,” Trans. Roy. Soc. Edinb., Vol. 25, 1869, pp. 217-260.

[18] Lam, K. M., "Vortex Shedding Flow Behind a Slowly Rotating Circular Cylinder," Journal of Fluids and Structures, Vol. 25, No. 2, 2009, pp. 245-262.

[19] Mittal, S., and Kumar, B., "Flow Past a Rotating Cylinder," Journal of Fluid Mechanics, Vol. 476, No. 476, 2003 , pp. $303-334$.

[20] Melius, M. S., Mulleners, K., and Cal, R. B., "The Role of Surface Vorticity During Unsteady Separation," Physics of Fluids, 2018. 